\title{
Le passif en français et le statut référentiel du sujet
}

\author{
Pierre Jalenques \\ Université de Rouen, laboratoire Dysola \\ pierre.jalenques@univ-rouen.fr
}

\begin{abstract}
Résumé. Nous proposons de reprendre l'analyse du passif en français, appelé parfois passif périphrastique. Nous réexaminerons cet objet linguistique à partir de données généralement marginalisées dans son analyse, à savoir les lacunes distributionnelles par rapport à l'actif, comme : Cécile parle couramment l'anglais / ?? L'anglais est parlé couramment par Cécile; Marie a regardé la télé toute la soirée / ?? la télé a été regardée par Marie toute la soirée. Nous partirons de l'analyse proposée par P. Jalenques (2015) permettant de ramener ces contraintes à un principe régulier et expliquant ce dernier à partir de l'hypothèse que être n'est pas un simple auxiliaire mais correspond au verbe attributif. Nous montrerons que cette analyse est cependant incomplète et rend difficilement compte de lacunes comme les ouvriers ont cessé le travail / ?? le travail a été cessé par les ouvriers. En reprenant l'étude des contraintes sur les relations anaphoriques au passif, nous verrons qu'un second principe explicatif, tiré d'une hypothèse de E. Keenan (1976), vient compléter l'analyse de Jalenques (2015) et permet de rendre compte de la plupart des lacunes distributionnelles au passif.
\end{abstract}

\begin{abstract}
The passive in French and the refential status of the subject. We propose to resume the analysis of the passive construction in French, sometimes called passif périphrastique. We reconsider this linguistic object from data which usually receive little attention, namely the distributional gaps compared with the active construction, as: Cécile parle couramment l'anglais / ?? L'anglais est parlé couramment par Cécile ; Marie a regardé la télé toute la soirée / ?? la télé a été regardée par Marie toute la soirée. We start with the analysis proposed by P. Jalenques (2015), reducing these distributional constraints to a regular principle and explaining it by the assumption that etre is not a simple auxiliary but corresponds to the attributive verb. We will show that this analysis is however incomplete and doesn't account for distributional gaps like les ouvriers ont cessé le travail /?? le travail a été cessé par les ouvriers. By resuming the analysis of constraints on anaphoric relations in the passive, we shall see that a second explanatory principle, based upon a hypothesis of E. Keenan (1976), completes the analysis of Jalenques (2015) and allows to account for most of the distributional gaps in the passive construction.
\end{abstract}




\section{Introduction}

\subsection{Objet d'étude}

Nous nous proposons de reprendre l'analyse du passif en français, appelé parfois passif périphrastique ${ }^{1}$. Plus précisément, nous aborderons l'objet linguistique répondant aux trois caractéristiques suivantes :

i) dimension syntaxique :

- le second argument du verbe (noté Y) est en position de sujet syntaxique ;

- le premier argument (noté X) occupe une position syntaxiquement facultative ;

ii) dimension lexicale : présence de l'élément être

iii) dimension morphologique : le verbe suivant l'élément être apparaît sous la forme de participe passé.

$$
\text { une clôture électrique entoure la propriété }
$$

$$
\mathrm{X}
$$

la propriété est entourée par une clôture électrique

$\mathrm{Y}$

\section{$\mathrm{X}$}

Précisons d'emblée que nous ne considérons pas que le passif dériverait de la configuration active de quelque manière que ce soit. Il s'agit pour nous de deux objets linguistiques indépendants. La comparaison du passif avec l'actif ne vaut donc ici qu’à titre heuristique. Nous réexaminerons le passif à partir de données généralement marginalisées dans l'analyse de cet objet linguistique, à savoir les lacunes distributionnelles par rapport à l'actif. Considérons l'emploi suivant du verbe parler :

$$
\begin{aligned}
& \text { a. Cécile parle couramment l'anglais } \\
& \text { b. ?? L'anglais est couramment parlé par Cécile }
\end{aligned}
$$

Nous constatons que cet emploi du verbe parler est difficilement acceptable au passif. Ainsi, il ne suffit pas qu'un verbe du français ait un emploi en construction directe pour que cet emploi donne automatiquement lieu à un emploi au passif. Ce problème est bien connu et ces données ont été abordées à de nombreuses reprises dans la littérature (cf. notamment F. Brunot (1922), M. Gross (1975), N. Ruwet (1983), E. Keenan (1975), A. Siewierska (1984), C. Leclere (1993)).

Depuis les années 70, plusieurs hypothèses ont été proposées pour tenter de ramener tout ou partie de ces lacunes distributionnelles à un ou plusieurs principe(s) régulier(s). D. Gaatone (1998) les a reprises une à une et a relevé pour chacune d'elle des contre-exemples, parfois nombreux. Cela l'a amené à conclure que ces lacunes distributionnelles ont un caractère idiosyncrasique, irrégulier donc. 


\subsection{Problématique}

Si l'on conclut à la suite de Gaatone (1998) que ces lacunes distributionnelles correspondent effectivement à des idiosyncrasies, leur existence est sans conséquence sur l'étude du passif. Mais, si ces lacunes distributionnelles s'avéraient relever d'un ou plusieurs principes réguliers, alors cette ou ces régularités devraient nécessairement être corrélées à des hypothèses sur le passif, puisque ces lacunes s’observent au passif et non à l'actif.

Dans un article de 2015, P. Jalenques a repris l'étude de ces lacunes distributionnelles. A l'encontre de Gaatone, il soutient que ces données relèvent d'un principe régulier. Jalenques relève tout d'abord que les lacunes distributionnelles au passif sont généralement corrélées à la question de la transitivité définie sémantiquement comme une notion prototypique, gradable, à la suite des travaux de Hopper et Thompson $(1983)^{2}$. Concernant le passif, l'hypothèse est alors la suivante : le degré de passivabilité d'un emploi de verbe transitif direct est proportionnel à son degré de transitivité sémantique. En conséquence, les lacunes distributionnelles au passif s'expliqueraient par le caractère faiblement transitif des emplois correspondant à l'actif. Tout le problème est que cette corrélation a de nombreux contre-exemples. Considérons les deux emplois suivants du verbe séparer :

a. On a séparé les deux jumeaux à la naissance.

b. une mince cloison sépare les deux pièces

Le premier emploi correspond à un procès dynamique, agentif, mettant en jeu des participants animés humains. Le second emploi de ce même verbe correspond à un procès non agentif, statif, mettant en jeu des participants inanimés. Le second emploi est donc, sémantiquement, beaucoup moins transitif que le premier. Il est pourtant tout aussi passivable que le premier :

a. les deux jumeaux ont été séparés à la naissance.

b. les deux pièces sont séparées par une mince cloison

Considérons encore les deux emplois suivants :

a. Marie a regardé la télé toute la soirée

b. un témoin anonyme a vu le directeur en compagnie du suspect

On oppose traditionnellement les verbes regarder et voir par le trait d'agentivité, le premier verbe étant agentif, le second étant non agentif. Le verbe regarder est donc plus proche du prototype de transitivité que voir. Pourtant, à la suite de Gaatone (1998), on observe que le verbe regarder est plus difficilement passivable que voir :

a. ?? la télé a été regardée toute la soirée par Marie ${ }^{3}$

b. le directeur a été vu en compagnie du suspect par un témoin anonyme

Considérons enfin les deux emplois suivants du verbe parler, le premier reprenant l'exemple (3) :

a. Cécile parle couramment l'anglais

b. On parle l'anglais dans le monde entier

Dans ces deux emplois le sens du verbe est le même et les rôles sémantiques des participants au procès sont les mêmes; le degré de transitivité sémantique de ces deux emplois du verbe parler est donc similaire dans les deux cas. Pourtant, seul le second donne une séquence passive acceptable :
a. ?? l’anglais est parlé couramment par Cécile
b. l' anglais est parlé dans le monde entier 
Ainsi le degré d'acceptabilité d’une séquence au passif n’est pas proportionnel au degré de transitivité sémantique de cette séquence. En reprenant l'analyse sémantique des contraintes distributionnelles, Jalenques (2015) montre que, dans tous les cas, le passif impose que le référent du second argument du verbe, Y (le sujet du passif), soit caractérisé par les autres éléments de la relation prédicative, indépendamment du type de procès associé au verbe, et indépendamment des rôles sémantiques des arguments du verbe. Ce principe régulier découle de l'hypothèse selon laquelle le passif est constitué du verbe être (qui n'est donc pas qu'un simple auxiliaire vide de sens). Dans cette hypothèse, le passif est un cas particulier de construction attributive ${ }^{4}$.

Revenons à l'exemple (8). En (8a), le procès caractérise le premier participant Cécile (elle est compétente en anglais) et non le second la langue anglaise (le fait que Cécile parle l'anglais ne suffit pas à définir une caractéristique de cette langue). Au contraire, en (8b), la présence du complément dans le monde entier induit sémantiquement une caractérisation de la langue anglaise (elle est très répandue, parlée par beaucoup de locuteurs, etc.), donc une caractérisation du sujet du passif, contrainte imposée par le verbe être. L'explication de la différence d'acceptabilité entre (8a) et (8b) ne découle pas d'une différence de degré de transitivité dans ces emplois du verbe parler ; elle découle de leur différence de compatibilité avec la dimension attributive du verbe être. Nous renvoyons à Jalenques (2015) pour une analyse développée de ce type d'emplois.

Il subsiste cependant deux problèmes. Premièrement, bien que cette analyse permette de régulariser un certain nombre de données, elle ne paraît pas pouvoir rendre compte de l'ensemble des lacunes distributionnelles. En effet, considérons les deux emplois suivants :

$$
\begin{aligned}
& \text { a. l'inspecteur allongea le pas } \\
& \text { b. les ouvriers ont cessé le travail }
\end{aligned}
$$

Ces deux emplois évoquent un changement de situation qui a un effet sur le second argument du verbe : en (10a), on comprend que les pas sont plus grands, en (10b) on comprend que le déroulement du travail est arrêté. A priori donc, ces deux emplois vérifient la contrainte du passif mise au jour par Jalenques : le second argument est interprété comme caractérisé par l'actualisation du procès. Cependant, ces deux séquences ne sont pas passivables :

$$
\begin{aligned}
& \text { a. * le pas a été allongé par l’inspecteur } \\
& \text { b. * le travail a été cessé par les ouvriers }
\end{aligned}
$$

Il apparaît que certaines séquences au passif, satisfaisant à première vue la contrainte générale mise au jour dans Jalenques (2015), sont difficilement passivables.

Deuxièmement, l'analyse de Jalenques ne prend pas en considération les contraintes relevant du domaine de l'anaphore. Or, il est bien connu que lorsqu'il y a une relation anaphorique entre le sujet du passif et le complément d'agent, l'emploi du passif est difficile (nous reprenons ces exemples à Gaatone) :

- domaine grammatical (anaphore possessive) :

a. Marie a retrouvé sa montre

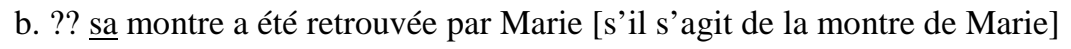

- domaine des relations lexicales (anaphore associative):

$$
\begin{aligned}
& \text { a. à la bataille, } \underline{\text { Paul a perdu un bras }} \\
& \text { b. ?? à la bataille, un bras a été perdu par Paul [s’il s’agit du bras de Paul] }
\end{aligned}
$$

Cependant, concernant les contraintes au passif sur les relations d'anaphore associative, Gaatone conteste leur généralité en relevant plusieurs contre-exemples de séquences attestables avec un nom de partie du corps, comme : 
(14) quand toutes les mains seront levées, le chef pourra être sûr de l'appui unanime

Nous nous proposons donc de rouvrir le dossier concernant ces contraintes distributionnelles. Dans un premier temps, nous reprendrons l'analyse des contraintes sur les relations d'anaphore associative au passif. Nous verrons que les contre-exemples de Gaatone peuvent être résolus. Dans un second temps, nous verrons que l'explication des contraintes sur les relations anaphoriques permettent de rendre compte des lacunes distributionnelles illustrées en (10) en mobilisant un second principe régulier, reprenant une hypothèse de E. Keenan (1976). Ce second principe ne contredit pas l'analyse de Jalenques (2015) mais vient la compléter.

\subsection{Positionnement par rapport à des débats non abordés ici}

Avant d'aborder la première partie, précisons quelques points généraux sur le passif. On le sait, l'étude du passif est le lieu de multiples débats entre cadres théoriques et parfois à l’intérieur des cadres théoriques. Nous ne saurions les reprendre tous ici. Ceci dit, pour limiter autant que possible les malentendus éventuels dans la suite de l'article, précisons trois d'entre eux.

Un premier débat concerne le statut du complément d’agent. On peut distinguer deux grandes tendances opposées. Dans la première tendance, les auteurs affirment que le passif correspond à la perte d'un argument pour le verbe, en l'occurrence la perte de son premier argument (celui qui correspond au complément d'agent) ${ }^{5}$; nous aurions donc une réduction de la valence du verbe. Dans cette hypothèse, le complément d'agent ne correspondrait pas à un argument du verbe, mais aurait un rôle proche de celui de complément circonstanciel. Il s’agit de l'analyse classiquement défendue en grammaire générative (cf. Helland, 2002). Dans la seconde tendance, les auteurs considèrent que la valence du verbe ne change pas au passif ; le complément d'agent est bien un argument du verbe (son premier argument). Cependant, par différence avec l'actif, ce premier argument est mis à l'écart de la relation prédicative, marginalisé, d'une façon ou d'une autre. C'est le point de vue défendu par exemple dans C. Muller (2000). Notre analyse du passif se situe dans cette seconde tendance.

Un second débat concerne le statut du participe passé au passif. Un certain nombre d’approches considèrent que cet objet linguistique a des propriétés spécifiques qui le distinguent du participe passé aux temps composés de l'actif (passé composé, etc.) et dans la configuration pronominale. Certains auteurs parlent ainsi de "participe passé passif ». Cette analyse revient à considérer que les participes passés à l'actif et au passif sont des homonymes (cf. Langacker, 1991 : 201 ; Helland, 2002 : 45). Cette thèse homonymique du participe passé pose de nombreux problèmes ; mais la thèse inverse admettant que le participe passé est le même objet linguistique à l'actif et au passif pose également des problèmes de cohérence à notre connaissance non résolus. Ce débat excède donc largement les limites de notre article. Nous ne prendrons donc pas position sur ce point. Nous parlerons simplement de "participe passé », au passif, sans préjuger de ses liens éventuels avec le participe passé à l’actif.

Un troisième débat concerne la distinction éventuelle entre deux passifs périphrastiques : un passif verbal et un passif adjectival (cf. Helland, 2002). En l'occurrence, la distinction entre ces deux sous-types de passifs périphrastiques repose centralement sur l'opposition aspectuelle entre procès dynamiques (passif verbal) et procès statifs (passif adjectival). Dans la mesure où les propriétés que nous mettrons au jour sont communes à ces deux types de procès, la distinction n'est pas opératoire dans la présente analyse. Nous parlerons donc simplement du passif (périphrastique). Venons-en maintenant au réexamen des relations anaphoriques dans le cadre du passif. 


\section{Passif périphrastique et anaphore associative}

Avant de reprendre l'analyse des contraintes sur les relations d'anaphore associative au passif, il convient de préciser quelques points.

\subsection{Quelques données concernant l'anaphore associative}

On entend habituellement par anaphore indirecte une relation anaphorique entre deux expressions dont les éléments désignés sont non coréférentiels ${ }^{6}$. Parmi les anaphores indirectes, une classe importante est constituée par les anaphores associatives qui sont souvent présentées comme mettant en jeu des relations de type partie-tout comme dans l'exemple suivant repris à Kleiber (2001 : 9) :

(15) Les policiers inspectèrent la voiture. Les roues étaient pleines de boue

$$
\text { antécédent }
$$

La définition même de l'anaphore associative donne lieu à de nombreux débats, certains auteurs l'identifiant à l'ensemble des anaphores indirectes ${ }^{7}$. Ce débat ne nous concerne pas directement. Ce qu'il importe de prendre en compte ici, qu'il s'agisse d'anaphore associative au sens étroit ou large, c'est le fait général que dans une relation d'anaphore indirecte, l'existence du référent d'une expression est rapportée à l'existence du référent d'une autre expression (l'antécédent). Dans l'exemple (15) cidessus, l'existence du référent de les roues est rapportée à l'existence du référent de la voiture.

L'autre élément crucial pour notre analyse concerne l'orientation sémantique de la relation anaphorique : étant donné une expression pouvant désigner un tout, et une expression pouvant désigner une partie, lequel des deux éléments constitue l'antécédent de l'autre, puisqu'aucune marque grammaticale ne précise l'orientation sémantique de la relation ? A priori, linguistiquement, on pourrait supposer que la relation n'est pas orientée et que selon les contextes, c'est l'une ou l'autre expression qui pourrait constituer l'antécédent. Or, il n’en est rien.

Kleiber (2001 : 182-202) montre que, de façon générale, l’antécédent est constitué par l'expression désignant le tout par rapport à l'expression désignant une partie de ce tout. Certes, sur l'axe syntagmatique, on peut avoir des cas où l'expression désignant la partie précède l'expression désignant le tout :

$$
\text { les pneus étaient lisses. La voiture a dérapé }
$$

Mais cette précédence syntagmatique n’implique pas une précédence sémantique où la partie constituerait l'antécédent. Kleiber (2001 : 193) souligne que « le changement d'ordre n’entraîne en effet pas de changement dans l'interprétation de la dépendance interprétative que la partie soit placée avant ou après le tout, elle est dans le même rapport interprétatif vis-à-vis du tout. C'est elle qui se trouve déterminée par le tout et non l'inverse ». Sans reprendre l'ensemble de sa démonstration, notons l'argument suivant : même lorsque le SN désignant le tout est placé après le SN désignant la partie, l'identification de celui-là n'est pas fondée à partir de celui-ci. Cela peut être montré à partir d'une question sur l'identification du référent du tout ou bien du référent de la partie (exemple repris à Kleiber (id.) :

$$
\begin{aligned}
& \text { - les pneus étaient lisses. La voiture a dérapé } \\
& \text { - quelle voiture? }
\end{aligned}
$$




\author{
- les pneus étaient lisses. La voiture a dérapé \\ - ?? quels pneus ? [dans le cas où les pneus désignent l’ensemble des pneus de la \\ voiture]
}

L’incongruité de la question en (18) par différence avec celle de (17) montre que l'identification du référent les pneus est bien fondée à partir du référent de l'expression la voiture et non l’inverse.

En résumé, lorsque dans une relation prédicative nous avons deux expressions en relation anaphorique indirecte, où l'une représente un tout par rapport à l'autre qui représente une partie ou un ingrédient de ce tout, alors l'expression désignant le tout correspond à l'antécédent, et l'expression désignant la partie ou l'ingrédient correspond à l'expression anaphorique. Nous pouvons maintenant aborder les séquences au passif.

\title{
2.2. Le passif et l'anaphore associative
}

\subsubsection{Quelques remarques préliminaires sur l'anaphore grammaticale au passif}

Comme nous l'avons rappelé en introduction, les contraintes sur les relations anaphoriques grammaticales au passif sont bien connues ${ }^{8}$. D'un auteur à l'autre, cela tourne toujours autour de l'idée de coréférence : « il y a toutes sortes de phrases inacceptables au passif, pour diverses raisons. Pour des raisons de coréférence tout d'abord » (Lamiroy, 1993 : 58). On retrouve la même idée dans A. Siewierska (1984: $207)$ : «It is not thus simply the presence of a possessive pronoun which "disallows" the passive, but rather coreference in general ». Herslund (1996 : 39) donne une formulation plus précise : « le passif est exclu, ou problématique, s'il existe une relation de coréférence entre le sujet et le complément d'agent ».

Cependant, ainsi formulée, la contrainte sur les relations anaphoriques au passif a manifestement des contre-exemples. Gaatone (1998) donne l'exemple attestable suivant où le sujet et le complément d'agent sont en relation de coréférence. Selon la notation habituelle, nous marquons par un indice identique «i»le lien d'anaphore entre l'expression anaphorique et son antécédent et par des indices différents «i » et «k» l'absence de lien anaphorique.

$$
\text { Mais } \frac{\text { Napoléon }_{\mathrm{i}}[\ldots] \text { a été trahi par lui-même }}{\mathrm{Y}} \text {. }
$$

Il ne suffit pas de prendre en compte l'existence d'une relation anaphorique entre les arguments du verbe ; il faut également prendre en compte l'orientation syntaxique de cette relation anaphorique. En (19), c'est le premier argument X (lui-même) qui réfère au second argument Y Napoléon. Or, il suffit d'inverser les positions syntaxiques des arguments en relations anaphoriques pour constater à nouveau le blocage du passif :

(20) * lui-même $\mathrm{i}_{\mathrm{i}}$ a été trahi par Napoléon $_{\mathrm{i}}$

Par contre, il n’y a pas de problème si lui-même n’a pas de lien anaphorique avec Napoléon :

(21) lui-même ${ }_{\mathrm{i}}$ a été trahi par Napoléon $_{\mathrm{k}}$

C'est donc l'orientation syntaxique de la relation anaphorique qui est cruciale dans l'émergence d'un blocage ou pas dans la configuration passive lorsqu'il y a coréférence entre les arguments. Au passif, on ne peut pas avoir l'expression anaphorique en position de sujet et l'antécédent en position de complément d'agent.

Mais, cette contrainte n’est pas spécifique au passif. Elle s’observe également avec la diathèse active, même si elle peut sembler intuitivement moins nette qu'au passif dans le cas du déterminant possessif : 


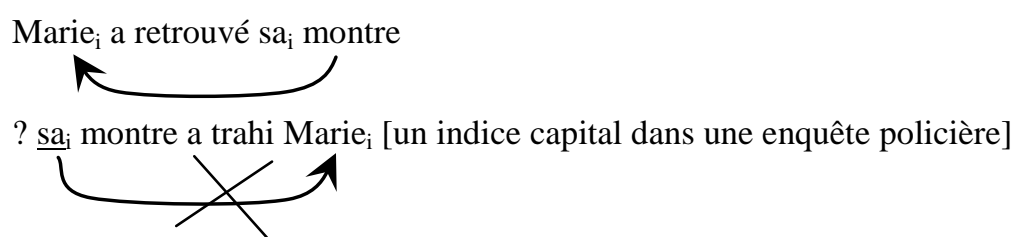

Néanmoins, de façon régulière, avec différents verbes, on obtient des séquences peu naturelles dans la même configuration anaphorique en diathèse active :
a. ?? son $_{\mathrm{i}}$ fils a étonné Paul
b. ?? $\operatorname{son}_{\mathrm{i}}$ chien a mordu $\mathrm{Paul}_{\mathrm{i}}$

Cette contrainte sur l'orientation syntaxique de la relation anaphorique découle de la position syntaxique de sujet de l'élément anaphorique par rapport à son antécédent en position de complément. Ce type de contraintes a été largement abordé dans les travaux en grammaire générative en d'autres termes. Sans entrer dans les détails et dans les explications formelles, on considère classiquement dans ce cadre qu'une expression anaphorique ne peut pas c-commander son antécédent, du moins lorsque ce dernier correspond à une expression référentielle (cf. Zribi-Hertz, $1996: 56)^{9}$. Ce type d’hypothèses a été largement débattu dans le cadre plus général de la théorie du liage, elle-même fortement débattue ${ }^{10}$.

Peu importe ici le détail. Il nous importe de constater que nous retrouvons au passif une contrainte générale sur les relations anaphoriques pronominales concernant le sujet syntaxique et le complément d'agent, existant indépendamment de la configuration passive. Revenons maintenant aux relations d'anaphore associative.

\subsubsection{Le passif et l'anaphore associative}

Nous partirons d'une observation que Gaatone (1998 : 64) reprend à N. Ruwet (1983 : 127), à savoir que les prédicats dont les arguments sont en relation sémantique de partie-tout se passivent difficilement, en particulier lorsque la partie correspond à un nom de partie du corps ${ }^{11}$. Notons par un indice identique «i» le lien d'anaphore entre le nom désignant la partie et le nom antécédent désignant le tout ; notons par des indices différents «i ${ }_{\text {i }}$ et «k l'absence de lien anaphorique ${ }^{12}$.

$$
\begin{gathered}
\text { a. à la bataille, } \underline{\text { Paul }}_{i} \text { a perdu un bras } \\
\text { antécédent }
\end{gathered}
$$

b. ?? à la bataille, $\underline{\underline{u n} \text { bras }_{i}}$ a été perdu par $\underline{\operatorname{Paul}}_{i}$

$$
\text { antécédent }
$$

Précisons d'emblée que le problème ne se trouve pas au niveau du déterminant du sujet du passif, puisque le passif ne marche pas même si l'on a un déterminant défini :

$$
\begin{aligned}
& \text { a. François }{ }_{i} \text { a baissé les yeux } x_{i} \\
& \text { b. ?? les yeux } x_{i} \text { ont été baissés par François } s_{i}
\end{aligned}
$$

Reprenons l'exemple (25). Imaginons un contexte où il ne s'agit pas du bras de Paul. Par exemple, imaginons une usine de fabrication d'androïdes. Paul, un technicien, devait apporter une série de bras dans le hall de montage des robots. Dans ce contexte, la séquence est attestable :

$$
\text { c. } \frac{\text { un bras }}{\mathrm{Y}} \mathrm{a} \text { a été perdu par } \frac{\mathrm{Paul}_{\mathrm{k}}}{\mathrm{X}}
$$


On pourrait en déduire que le passif bloque lorsqu'il y a une relation d'anaphore indirecte entre les deux arguments du verbe. Mais l'exemple suivant montre qu'il n'en est rien :

$$
\text { à son réveil, } \frac{\mathrm{Paul}_{\mathrm{i}}}{\mathrm{Y}} \text { a été effrayé par son bras } \frac{\text { qui avait doublé de volume }}{\mathrm{X}}
$$

Quand les positions syntaxiques sont inversées et que le SN correspondant à l'antécédent (désignant le tout) est en position sujet, alors l'emploi du passif ne pose pas de problème. Donc, là encore, comme dans le cas de coréférence entre les deux arguments du verbe, ce qui est en jeu, c'est l'orientation syntaxique de la relation d'anaphore associative entre les arguments du verbe et non l'existence même d'une relation anaphorique entre ces arguments. L'emploi du passif marche mal lorsque l'expression anaphorique (le nom désignant la partie) est en position de sujet syntaxique et l'antécédent (le nom désignant le tout) en position de complément d’agent, ce qui est le cas en (25b) :

a. à la bataille, $\underline{P a u l}_{\underline{i}}$ a perdu un bras $_{\mathrm{i}}$

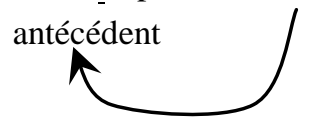

b. ?? à la bataille, un bras $_{i}$ a été perdu par $\underline{\operatorname{Paul}}_{i}$

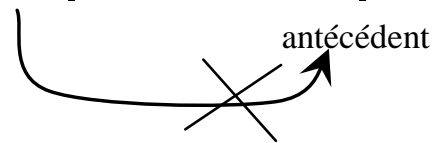

Mais cette contrainte ne semble pas spécifique au passif. On la retrouve en effet à l’actif. Considérons la séquence suivante :

$$
\text { ?? } \underline{\text { un bras }}_{\underline{i}} \text { fait souffrir } \underline{\text { Paul }}_{i} \underline{13}
$$

Dans cette séquence, l'anaphorisant est en position syntaxique de sujet et son antécédent, inclus dans le $\mathrm{SV}$, correspond à une expression référentielle ; comme au passif, nous constatons que la séquence est difficilement acceptable. Par contre, si l'antécédent de l'argument en position sujet est un pronom clitique (expression non référentielle), alors la relation anaphorique ne pose pas de problème, comme pour l'anaphore pronominale :

$$
\begin{aligned}
& \text { a. le bras } \text { gauche la }_{\mathrm{i}} \text { démange } \\
& \text { b. ses } \mathrm{s}_{\mathrm{i}} \text { lunettes } \mathrm{la}_{\mathrm{i}} \text { gênent }
\end{aligned}
$$

Sous réserve d'analyses complémentaires venant confirmer ces dernières observations, il semble donc que la contrainte sur l'orientation syntaxique de la relation d'anaphore associative entre les arguments du verbe ne soit pas spécifique au passif mais constitue une contrainte générale liée à la position syntaxique de sujet, lorsque l'antécédent en position de complément constitue une expression référentielle. Nous retrouvons donc au niveau lexical (anaphore associative) la même contrainte générale que celle manifestée au niveau grammatical (anaphore pronominale) et cette contrainte semble non spécifique à la configuration passive.

Ceci étant précisé, Gaatone (1998) fournit des données qui constituent a priori des contre-exemples à ce principe général. L’auteur présente les cinq séquences suivantes où le second argument du verbe $(\mathrm{Y})$ en position de sujet renvoie à un nom de partie du corps, constituant donc a priori une expression anaphorique liée au complément d'agent : 

a. La main droite levée, le témoin jure de dire la vérité
b. Quand toutes les mains seront levées, le chef pourra être sûr de l'appui unanime
c. La main lui a été coupée (par le bourreau)
d. Une fois sa colère passée sur ses proches, il se calma
e. Des poings ont été brandis par les manifestants

Notons que dans deux de ces séquences (32a,d), le verbe être est absent. Mais surtout, dans les quatre premières séquences il n’y a pas de complément d'agent. Dans (32a,b,d) le terme correspondant sémantiquement au complément d'agent est exprimé dans une seconde proposition. En (32c), le complément d'agent (le bourreau) n'est pas l'antécédent anaphorisé de main. Si on rétablit le verbe être et un complément d'agent antécédent du sujet là où ils sont absents, les séquences obtenues sont peu acceptables :
a. ?? La main droite a été levée par le témoin
b. ? Quand toutes les mains seront levées par les participants, ...
c. ?? La main $n_{\mathrm{i}}$ a été coupée par Paul $\mathrm{i}_{\mathrm{i}}$
d. ?? Une fois que $\mathrm{sa}_{\mathrm{i}}$ colère a été passée par Paul $\mathrm{i}_{\mathrm{i}}$ sur ses proches, il se calma

Seule la dernière séquence (32e) fournie par Gaatone comporte un complément d'agent correspondant sémantiquement à l'antécédent du second argument du verbe des poings. Mais cet emploi est très contraint. Tout d’abord, l'auteur reconnaît qu'avec le déterminant défini, la séquence n’est guère attestable :

$$
\text { ?* Les poings ont été brandis par les manifestants }
$$

Pour dépasser le recours à la seule intuition, nous avons regardé sur corpus les cooccurrences de brandir avec poing(s), au passif. Dans le corpus FrWac de SketchEngine, nous relevons 113 occurrences du verbe brandir au passif ${ }^{14}$, dont seulement 21 avec un complément d'agent (soit 18,5\% des emplois). Ce verbe est donc d'emploi peu fréquent avec un complément d'agent. Ceci dit, nous ne relevons dans ce corpus aucune occurrence de passif du verbe brandir avec le nom poing(s). Nous avons donc élargi la recherche à l'internet francophone dans son ensemble (recherche sur google ; novembre 2015). Nous avons relevé 42 occurrences $^{15}$. Ces 42 séquences sont toutes sans complément d'agent, sauf une :

Avant de venir à la parole, les mains et surtout les poings étaient brandis par nos ancêtres pour défendre leurs familles ou territoires.

Dans cette séquence, le statut référentiel de poings est particulier. Il a une autonomie que n’a pas le mot main dans Paul a levé la main. Ici, poings a une signification symbolique et se rapproche de termes comme slogan / revendications / etc. En dehors de ce cas particulier, les séquences attestées avec poings correspondent à des passifs sans agent exprimé :

\section{a. Enfin, Des mains frappent, des poings sont brandis}

b. Dans une violence pure, des poings sont brandis, des os sont fracassés et l'antipathie est palpable.

c. 5 mai 2006 - Si la situation ne s'améliore pas, le ton monte, des doigts sont pointés et des poings sont brandis.

Par ailleurs, indépendamment du nom poing, nous avons observé les termes en position de sujet dans les emplois au passif de brandir. La plupart des arguments sujets correspondent à des termes comme drapeau, banderole, portrait, slogan, etc., c'est-à-dire des termes qui ne sont pas dans une relation anaphorique au complément d'agent. Contrairement à ce que l'on a observé avec poing(s), on relève avec ces noms de nombreux exemples présentant un complément d'agent : 

a. Comme la veille, beaucoup de pancartes et stylos ont été brandis par la foule.
b. de très nombreux messages de soutien envers les réfugiés ont été brandis par les supporters
c. Des posters de M. M. ont été brandis partout par les manifestants

Donc, lorsque le sujet du passif n’anaphorise pas le complément d'agent, sa présence ne pose pas de problème ainsi que le montre les séquences attestés ; par contre lorsque le sujet syntaxique du passif anaphorise le complément d'agent, ce dernier est absent dans les emplois attestés ou bien d'emploi très contraint.

En conclusion, les données fournies par Gaatone en (32) ne constituent pas des contre-exemples à la contrainte sur l'orientation syntaxique de la relation anaphorique associative entre les arguments du verbe. Lorsque le complément d'agent constitue l'antécédent anaphorique du second argument du verbe (le complément d'agent), alors la séquence au passif est peu naturelle.

Nous faisons l'hypothèse que l'absence du complément d'agent dans le cas d'un sujet du passif avec un nom de partie du corps est un moyen de contourner la contrainte sur la relation anaphorique entre les arguments du verbe au passif lorsqu'elle est orientée du second argument du verbe (sujet du passif) vers le premier argument (complément d'agent). Ceci dit, même sans complément d'agent, l'emploi d'un nom de partie du corps en position de sujet syntaxique du passif est extrêmement contraint. Par exemple, on ne trouve sur google aucune occurrence de la séquence jambe a été tendue.

\subsection{Généralisation : I'hypothèse de E. Keenan (1976)}

La régularité de la contrainte sur les relations d'anaphore associative étant établie, celle-ci peut être vue comme un cas particulier d'une contrainte sémantique générale associée à la position syntaxique de sujet. En effet, E. Keenan (1976 : 312-313) établit comme première propriété fondamentale associée à la position de sujet syntaxique la propriété d'autonomie. Celle-ci a deux aspects:

a) "Independent Existence. The entity that a b-subject ${ }^{16}$ refers to (if any) exists independently of the action or property expressed by the predicate" (id. p. 312);

b) "Autonomous Reference. The reference of a b-subject must be determinable by the addressee at the moment of utterance. It cannot be made to depend on the reference of other NPs which follow it." (id. p. 313).

L'entité désignée par l'argument en position sujet est donc référentiellement indépendante à la fois par rapport au procès et par rapport à un autre argument du verbe (le premier argument dans le cas qui nous intéresse ici. Or, la relation sémantique entre une expression anaphorique et son antécédent correspond fondamentalement à une relation de dépendance référentielle, dans la mesure où l'entité désignée par l'expression anaphorique est référentiellement liée à l'entité désignée par l'antécédent. D’après l'hypothèse de Keenan, dès lors qu'une expression anaphorique occupe la position syntaxique de sujet, elle ne peut plus être référentiellement dépendante du complément d'agent. Ce dernier ne peut donc pas constituer son antécédent.

À notre connaissance, cette hypothèse sémantique associée à la position syntaxique de sujet n'est généralement pas prise en compte dans les études consacrées au passif, en particulier pour le français. Certes Keenan lui-même associe ce principe à une hypothèse sur le passif ( « in the passive we require that the referent of the object NP be indentifiable independently of that of the agent » (id., p. 310)), mais il ne la développe pas et n’aborde pas les données que nous allons examiner ici. De plus, il ne développe pas cette hypothèse dans ses travaux ultérieurs consacrés au passif (cf. en particulier E. Keenan et M. Dryer (2006)). Nous allons voir ci-après qu'elle constitue pourtant le principe explicatif fondamental permettant de rendre compte des données évoquées en (10). 


\section{Retour sur les lacunes distributionnelles relevant de la sémantique des verbes}

Reprenons maintenant l'examen des lacunes distributionnelles du type évoqué en (10). Reprenons l'exemple suivant avec le verbe cesser :

$$
\begin{aligned}
& \text { a. Les ouvriers ont cessé le travail } \\
& \mathrm{X} \\
& \text { b. ?? le travail a été cessé par les ouvriers } \\
& \mathrm{Y}
\end{aligned}
$$

Pour expliquer l'inattestabilité de (38b), la propriété proposée par Jalenques (2015), à savoir que la relation prédicative doit caractériser $\mathrm{Y}$, ne suffit pas. Ici, il y a bien l'idée que le travail est arrêté, le procès est donc a priori interprétable comme caractérisant Y. Ce qui est crucial ici, c'est la nature de la relation référentielle entre les deux arguments du verbe $\mathrm{Y}$ et $\mathrm{X}$. En effet, si on propose un contexte où travail est rapporté à un autre support que $\mathrm{X}$, on constate que l'emploi de cesser est impossible, par différence avec des verbes comme interrompre, stopper ou bloquer :
a. les manifestants ont interrompu le travail de la commission
b. les manifestants ont stoppé le travail de la commission
c. les manifestants ont bloqué le travail de la commission
d. * les manifestants ont cessé le travail de la commission

Ce différentiel d'acceptabilité montre qu'avec cesser le terme travail est nécessairement rapporté au premier argument du verbe ; plus précisément, cela signifie que l'existence même du référent de travail est rapporté au référent de ouvrier ; autrement dit, $\mathrm{X}$ est le support de l'existence de $\mathrm{Y}$. On constate que les trois autres verbes qui n'expriment pas cette contrainte sont passivables :

(40) le travail de la commission a été interrompu/stoppé/bloqué par les manifestants

Bien que ces quatre verbes soient sémantiquement proches, cesser s'en distingue par le fait qu'il impose, de par son sens même, une relation de dépendance référentielle entre les référents de ses arguments. Dans la mesure où, au passif, le second argument occupe la position syntaxique de sujet, il ne peut pas être dans une relation de dépendance référentielle au complément d'agent. Il y a donc une contradiction entre la contrainte sémantique imposée par le sens du verbe cesser et la contrainte sémantique associée à la position syntaxique de sujet ; d’où le caractère inattestable de (39d).

Prenons deux autres exemples qui $\mathrm{v}^{\wedge} \wedge$ ont nous permettre de préciser ce point. Comparons deux emplois statifs sémantiquement proches du verbe comprendre, dont l'un est passivable et l'autre difficilement. Ces exemples sont inspirés de ceux proposés par Gaatone (id., p. 106-107) :

a. le prix comprend les frais d'envoi du DVD

b. les frais d'envoi du DVD sont compris dans le prix

a. ma thèse comprend une partie sur l'aspect lexical

b. ?? une partie sur l'aspect lexical est comprise dans ma thèse

A première vue, on ne voit pas bien pourquoi seul (41b) serait passivable étant donné que le sens du verbe est très proche dans les deux cas. Pour (41a), Gaatone glose ce type d'emploi par la formulation «le complément d'objet direct désigne les éléments, les parties, qui composent l'entité représentés par le sujet »; puis il glose les emplois du type (42a) par « avoir en soi ». L'auteur ne propose pas d'explication du différentiel d'acceptabilité et celui-ci apparaît idiosyncrasique. 
Observons le statut référentiel du second argument. En (41), le procès exprime certes une relation d'inclusion de Y à X ; mais, cette relation d'inclusion ne concerne pas l'existence en soi de Y. En (42), c'est tout le contraire: à travers l'actualisation du procès, l'existence même du référent de $\mathrm{Y}$ est interprétée comme fondée à partir de l'existence de X. Cette différence peut être mise en évidence à l'aide de la négation :

$$
\begin{aligned}
& \text { a. le prix ne comprend pas les frais d'envoi du DVD } \\
& \text { b. ma thèse ne comprend pas de partie sur l'aspect lexical }
\end{aligned}
$$

La séquence (43a) ne signifie en aucun cas qu'il n’y a pas de frais d'envoi ; elle signifie qu'il y a bien des frais d'envoi mais qu'ils sont en plus du prix indiqué. Au contraire, la séquence (43b) signifie qu'il n’y a pas de partie sur l'aspect lexical ; la négation met en jeu l’existence même du référent de Y.

Ce qui est crucial, c’est donc l'autonomie référentielle du référent du second argument par rapport au premier argument. Le passif impose que l'existence du référent du second argument soit posée en soi, indépendamment de la relation au premier argument.

Examinons maintenant l'exemple suivant avec le verbe sentir :

$$
\begin{aligned}
& \text { a. le cuisinier sent le poisson } \\
& \text { b. le poisson est senti par le cuisinier }
\end{aligned}
$$

La séquence (44a) admet deux interprétations, selon les contextes où on l'insère : i) le cuisinier est en train de humer l'odeur d'un poisson ; ii), le cuisinier dégage une odeur de poisson. On constate qu'au passif seul la première interprétation est possible, ainsi que le révèle l'emploi d'un terme compatible uniquement avec la deuxième interprétation :

$$
\begin{aligned}
& \text { a. ta veste sent le poisson } \\
& \text { b. * le poisson est senti par ta veste }
\end{aligned}
$$

Cette différence d'acceptabilité est généralement considérée comme idiosyncrasique. Pourtant, là encore, on observe une différence cruciale entre les deux interprétations concernant le statut référentiel de Y : en i) on réfère à une situation où il y a un poisson; en ii), on réfère à une situation où il n'y a pas de poisson. Autrement dit, en i), le référent de $\mathrm{Y}$ est donné comme existant. En ii), il est donné comme n’existant pas ; plus précisément, son existence n'est pas dissociable de l'actualisation du procès (il n'est question de poisson qu'en tant que « poisson-senti », d'où l'interprétation qu'il y a une odeur de poisson mais pas forcément de poisson).

L'examen de cet exemple nous permet de préciser ce qui est en jeu dans la notion d'autonomie référentielle du second argument. Cette notion implique en fait une double contrainte :

\section{a) que Y ait une dimension référentielle (qu’il désigne en lui-même un référent) ;}

b) que la construction de la représentation de l'existence de ce référent soit fondée indépendamment de sa relation au premier argument.

Dès lors qu'une séquence ne satisfait pas la condition b) ou bien aucune des deux conditions, alors son emploi au passif donnera une séquence peu acceptable. Précisons que la contrainte selon laquelle Y doit avoir une dimension référentielle n'implique aucune restriction sur le type de catégories sémantiques auxquelles doit se rapporter le second argument du verbe. Prenons l'exemple d'un verbe métrologique, souvent donné comme exemple emblématique d'emploi non passivable :

$$
\begin{aligned}
& \text { a. leur bateau mesure } 12 \mathrm{~m} 50 \\
& \text { b. } * 12 \mathrm{~m} 50 \text { sont mesurés par leur bateau }
\end{aligned}
$$

Leclère (1993) souligne que ce type d'emplois n’est pas passivable car le verbe est employé dans un sens attributif ; or on sait que les verbes attributifs ne sont pas passivables. Cela est exact mais le constat de cette dimension attributive n'explique rien en soi. En effet, il reste à expliquer pourquoi les configurations 
attributives ne sont pas passivables. Cet emploi du verbe mesurer contredit simultanément deux contraintes du passif :

- celle selon laquelle la relation prédicative doit caractériser le second argument du verbe (or, ici elle caractérise le premier argument leur bateau);

- celle selon laquelle le second argument, en position sujet, doit avoir un référent autonome. Dans la mesure où $12 m 50$ désigne ici une propriété du bateau (correspondant à $\mathrm{X}$ ), Y n’est pas référentiellement indépendant de $\mathrm{X}$.

C'est le non-respect de ces deux contraintes qui rend cet emploi du verbe mesurer non passivable. Le problème ne réside pas dans la catégorie sémantique de Y lui-même, c'est-à-dire dans le fait qu'il exprime une quantité. En effet, dès lors que l'on a un contexte où cette quantité est envisagée en tant que tel, indépendamment de sa relation à un support, et que cette quantité est interprétée comme caractérisée par le procès, alors la séquence est passivable, comme dans les exemples attestés suivants :

a. Un arbre qui est destiné à mesurer plus de 2 mètres doit être planté à une distance minimum de 2 mètres de la limite de propriété (les $2 \mathrm{~m}$ sont mesurés de la limite de propriété à l'axe du tronc).

b. les $60 \mathrm{~m}$ sont mesurés à partir de la voie de desserte jusqu'à l'emprise constructible du terrain

Les mêmes observations et analyses peuvent être faites pour un verbe comme peser, ayant lui aussi un emploi statif métrologique :

$$
\begin{aligned}
& \text { a. ma voiture pèse } 800 \mathrm{~kg} \\
& \text { b.. *800 kg sont pesés par ma voiture } \\
& \text { [sur un forum internet] } \\
& \text { - quel est le poids à vide de ta Clio ? } \\
& \text { - Et le poids tractable? } \\
& \text { - Et tes } 800 \mathrm{~kg} \text { sont pesés ou estimés ? }
\end{aligned}
$$

On constate qu'avec un second argument désignant une quantité, et plus généralement des termes qui désignent une propriété, le premier argument du verbe sera généralement absent, pour satisfaire la contrainte d'autonomie référentielle du second argument (cf. les observations en (36) pour brandir le poing).

\section{Synthèse et bilan}

Il résulte de ce parcours que l'on peut rendre compte des lacunes distributionnelles au passif par la conjonction de deux contraintes générales :

A) le second argument doit pouvoir être interprété comme caractérisé par le prédicat associé au participe passé (ce prédicat inclut les modifieurs éventuels du Vpp)

B) le second argument du participe passé doit être référentiellement autonome, ce qui implique :

a) que $Y$ ait une dimension référentielle (qu’il désigne en lui-même un référent) ;

b) que la construction de la représentation de l'existence de ce référent soit fondée indépendamment de sa relation au premier argument.

Nous faisons l'hypothèse que ces deux contraintes générales sont toujours présentes dans tous les emplois au passif périphrastique. Dès lors qu'un emploi transitif direct d'un verbe ne satisfait pas l'une des 
contraintes, voire les deux, on obtient une séquence difficilement passivable. La prise en compte de ces deux contraintes permet en outre de rendre compte d'une hiérarchie stable chez les locuteurs dans le degré d’inacceptabilité des séquences difficilement passivables: certaines séquences jugées moyennement acceptables comme (3) contreviennent seulement à la première contrainte (emploi du verbe traverser) ; certaines séquences jugées totalement inacceptables contreviennent aux deux contraintes simultanément (emploi du verbe mesurer).

Ces deux contraintes sont transversales aux différents types de procès. Elles valent aussi bien pour rendre compte des séquences difficilement passivables avec les procès dynamiques qu'avec les procès statifs. Elles tendent à réinterroger la distinction traditionnelle entre un passif verbal et un passif adjectival. A tout le moins, elles soulignent une unité de fonctionnement entre les deux.

Aucun des éléments mis en jeu dans ces deux contraintes ne sont propres au passif périphrastique. Le fait que le sujet soit caractérisé par le prédicat (et ses éventuels modifieurs) est commun au passif et à la construction attributive du verbe être. Le fait que le sujet soit référentiellement autonome est commun au sujet du passif et au sujet de l'actif. Il apparaît donc que la spécificité du passif périphrastique réside dans la conjonction de ces différents éléments et non dans les éléments eux-mêmes. Ainsi, une analyse compositionnelle du passif périphrastique permet la mise au jour de régularités sémantiques qui excèdent la seule configuration passive. De ce point de vue, il semblerait que le passif périphrastique ne soit pas une construction, au sens des grammaires de construction, contrairement à l'hypothèse formulée dans Raineri (2010) ${ }^{17}$.

Enfin, cette analyse questionne les relations entre le passif et la transitivité. En effet, sur le plan sémantique, ces deux configurations syntaxiques sont fréquemment abordées en termes de prototype ; l'idée étant que les emplois prototypiquement transitifs seraient prototypiquement passivables. Or, comme nous l'avons vu, bien des emplois transitifs non prototypiques sont tout autant passivables que les emplois transitifs supposés prototypiques. L’analyse défendue ici revient à proposer des propriétés invariantes du passif communes à l'ensemble des séquences acceptables au passif. En ce sens, notre analyse du passif relève d'une sémantique non prototypiste ${ }^{18}$.

\section{Références}

ABEILLÉ, A. et GODARD, D. (2002). The syntactic structure of French auxiliaries, Language, 78:3, 404-452.

BRUNOT, F. (1922). La pensée et la langue. Paris : Masson et Cie.

DESCLES, J.-P. et GUENTCHEVA, Z. (1993). Le passif dans le système des voix du français. Langages, 109, 73102.

GAATONE, D. (1998). Le passif en français. Paris / Bruxelles : Duculot.

GOLDBERG, A. (1995). Constructions: A construction grammar approach to argument structure. Chicago : University of Chicago Press.

GOLDBERG, A. (2013). “Constructionist Approaches to Language”. In Thomas Hoffmann and Graeme Trousdale (eds.) Handbook of Construction Grammar, Oxford University Press.

GROSS, M. (1975). Méthodes en syntaxe. Régime des constructions complétives, Paris : Hermann.

HAMELIN, L. et LEGALLOIS, D. (sous presse), Une approche sémantique non prototypique de la construction transitive, Faits de langue, 45.

HELLAND, H. P. (2000). Le passif verbal et le passif adjectival. In L. Schösler (éd.), Le Passif, Actes du colloque de Copenhague 5-7 mars 1998, Etudes Romanes, 45, 83-97.

HELLAND, H. P. (2002). Le passif périphrastique en français moderne. Copenhague : Museum Tusculanum Press.

HERSLUND, M. (1996). Partitivité et possession inaliénable. Faits de langues, 7, 33-42.

HOPPER, P. et THOMPSON, S. (1980). Transitivity in Grammar and Discourse. Language, 56/2, 251-297. 
JALENQUES, P. (2010). Un exemple d'interaction morphologie-syntaxe-sémantique : les contraintes sur les procès transitionnels au passif périphrastique. Actes du VIII Congrès International de Linguistique Française, 25-27 septembre 2008, Université d’Oviedo, Liens linguistiques. Etudes sur la combinatoire des composants, Berne : Peter Lang, 409-425.

JALENQUES Pierre (2015). Le passif en français et les lacunes distributionnelles des verbes. Corela [En ligne], mis en ligne le 13-1 | 2015, (URL : http://corela.revues.org/4015).

KEENAN, E. L. (1976). Towards a universal definition of subject. In Ch. L. Li (ed.). Subject and Topic, New York / San Francisco / London : Academic Press, 305-333.

KEENAN, E. L. and DRYER M. S. (2006). Passive in the world's languages. In T. Shopen (ed.). Language Typology and Syntactic Description, Vol. 1, Clause Structure, second edition. Cambridge ; London : Cambridge university Press, 325-361.

KLEIBER, G. (2001): L’anaphore associative. Paris : PUF.

LAMIROY, B. (1993). Pourquoi il y a deux passifs. Langages, 109, 53-72.

LARREYA, P. (2012). Constantes et spécificités des procédés anaphoriques: étude contrastive de quelques marqueurs en français et en anglais. In C. Denizot et E. Dupraz (éds.), anaphore et anaphoriques, Mont-SaintAignan : Publications des universités de Rouen et du Havre, 15-30.

LECLERE, Ch. (1993). Classes de constructions directes sans passif. Langages, 109, 7-31.

LE GOFFIC, P. (1993). Grammaire de la Phrase Française. Paris : Hachette.

MULLER, Cl. (2000). Le passif processif et ses concurrents. Définition et quelques particularités. dans Le Passif, , Actes du colloque de Copenhague 5-7 mars 1998, L. Schösler (éd.), Etudes Romanes, 45, 49-69.

POSTAL, P. M. (1971). Cross-over Phenomena. New York : Holt, Rinehart and Winston.

RAINERI, S. (2010). Analyse contrastive français-anglais du passif dans une perspective constructionnelle : sens et fonction de BE Ven, ETRE Vé, GET Ven, SE FAIRE Ver, Thèse, Université Sorbonne Nouvelle - Paris 3.

RUWET, N. (1983). Du bon usage des expressions idiomatiques dans l'argumentation en syntaxe générative. Revue québécoise de linguistique, 13/1, 9-145.

SAFIR, K. (2004). The Syntax of Anaphora. Oxford Studies in Comparative Syntax.

SIEWIERSKA, A. (1984). The Passive. A Comparative Linguistic Analysis. London: Routledge.

ZRIBI-HERTZ, A. (1996). L'anaphore et les pronoms. Une introduction à la syntaxe générative. Villeneuve-d'Ascq : Presses universitaires du Septentrion.

\footnotetext{
${ }^{1}$ L'expression passif périphrastique est utilisée notamment par Gaatone (1998) et Helland (2002). Ici, nous parlerons simplement de passif.

${ }^{2}$ Pour un panorama sur la transitivité sémantique, nous renvoyons à J. François (1998).

${ }^{3}$ Précisons que la faible acceptabilité de cet emploi ne peut pas non plus s'expliquer par un quelconque degré d'idiomaticité puisque, comme le souligne Gaatone (1998), de nombreuses expressions idiomatiques sont parfaitement passivables (les points ont été mis sur les « $i$ » / la glace a été rompue entre les négociateurs / justice a été rendue, etc.).

${ }^{4}$ En soi, cette dernière hypothèse n'est pas nouvelle (cf. P. Le Goffic (1993), A. Abeillé et D. Godard (1996). Cependant, la spécificité de l'analyse de Jalenques est de fonder cette hypothèse à partir de l'analyse sémantique des contraintes distributionnelles au passif.

${ }^{5}$ Un des arguments classiques est le fait que le complément d’agent est généralement facultatif au passif, le caractère facultatif étant une des caractéristiques des compléments non argumentaux.

${ }^{6}$ Pour un bref panorama sur les relations anaphoriques, nous renvoyons à P. Larreya (2012).

${ }^{7}$ Pour une discussion sur la délimitation de ce qu’on entend par anaphore associative, cf. Kleiber (2001 : 8).
} 


\begin{abstract}
${ }^{8}$ Dans le cadre de la grammaire générative transformationnelle, ces contraintes ont été analysées à travers la notion de cross-over (cf. P. Postal (1971). Mais cette notion n'a de sens que dans le cadre d'une théorie syntaxique transformationnelle où le passif dérive syntaxiquement de l'actif. A l'heure actuelle, la plupart des analyses, $\mathrm{y}$ compris dans le cadre de la grammaire générative, considèrent que le passif est une configuration syntaxique indépendante de l'actif, point de vue que nous adoptons également. C'est pourquoi la notion de cross-over ne peut pas être utilisée ici.

${ }^{9}$ Il existe plusieurs versions de la relation de c-commande. Nous en donnons ici une version simple, suffisante pour les données qui nous intéressent, celle de Reinhart (1983), tirée de Zribi-Hertz (1996 : 56) : dans un arbre syntaxique, un nœud $\mathrm{A}$ c-commande un nœud $\mathrm{B}$ si a) $\mathrm{A}$ ne domine pas $\mathrm{B}$ et inversement ; b) le premier nœud à ramifications qui domine A domine également $\mathrm{B}$.
\end{abstract}

${ }^{10}$ Nous renvoyons à A. Zribi-Hertz, (1996) pour un panorama sur les contraintes syntaxiques concernant les relations anaphoriques pronominales en français. Pour une discussion générale plus récente, nous renvoyons à K. Safir (2004).

${ }^{11}$ J.-P. Desclès et Z. Guentchéva (1993 : 98) font la même observation.

${ }^{12}$ Précisons qu'ici, contrairement au cas d’anaphore grammaticale brièvement abordée précédemment, l’identité des indices marque une relation anaphorique sans coréférence. Il y a coindiciation mais pas de coindexation.

${ }^{13}$ Nous devons cet exemple à Laurent Gosselin (communication personnelle).

${ }^{14}$ Requête faite en novembre 2015 ; la requête concerne les séquences de la forme: est / était / a été / sont / étaient / ont été / brandi(e)(s)

${ }^{15}$ Requêtes : poing(s) est / sont / était / étaient / a été / ont été brandi(s).

16 "b-subject” renvoie à "basic-subject”, c'est-à-dire les sujets des phrases considérées comme des phrases de base sur le plan sémantique (cf. Keenan, 1976 : 306).

${ }^{17} \mathrm{Du}$ moins, dans l'acception la plus courante de la notion de construction en grammaire de construction : “a construction is posited in the grammar if it can be shown that its meaning and/or its form is not compositionally derived from other constructions existing in the language (A. Goldberg, 1995: p. 4). Plus récemment : "Constructions are defined to be conventional, learned form-function pairings at varying levels of complexity and abstraction. This definition is meant to highlight the commonality between words and larger phrasal units”. (A. Goldberg, 2013).

${ }^{18}$ Dans son principe, cette analyse rejoint la démarche de L. Hamelin et D. Legallois (sous presse) consacrée à une analyse sémantique non prototypiste de la construction transitive directe en français. 